\title{
Supporting information for \\ Epitaxial Intercalation Growth of Scalable Hexagonal Boron Nitride/Graphene Bilayer Moiré Materials with Highly Convergent Interlayer Angles
}

Shengnan Wang, * † Jack Crowther, †Hiroyuki Kageshima, § Hiroki Hibino, " and Yoshitaka Taniyasu ${ }^{\dagger}$

$\dagger$ NTT Basic Research Laboratories, NTT Corporation, Atsugi, Kanagawa 243-0198, Japan

$\S$ Graduate School of Natural Science and Technology, Shimane University, Matsue, Shimane 690-8504, Japan

"Department of Nanotechnology for Sustainable Energy, School of Science and Technology, Kwansei Gakuin University, Sanda, Hyogo 669-1337, Japan

*Correspondence and requests for materials should be addressed to S.W. (shengnan.wang.dy@hco.ntt.co.jp)

Fig. s1. Schematic illustration of the furnace tube for the growth of $\mathrm{hBN} / \mathrm{G}$ bilayers.

Fig. s2. Simulation of gas distribution in CVD furnace with a one-end-closed inner tube.

Fig. s3. SEM images of hBN grown with or without the inner tube.

Fig. s4. Timeline of CVD growth of $\mathrm{hBN} / \mathrm{G}$ on $\mathrm{Cu}$ catalyst.

Fig. s5. SEM images of as-grown hBN/G heterostructure film with different annealing steps.

Fig. s6. SEM image of adlayer growth of graphene under hBN with full coverage on $\mathrm{Cu}$.

Fig. s7. XPS survey spectra of $\mathrm{hBN} / \mathrm{Cu}$ and $\mathrm{hBN} / \mathrm{G} / \mathrm{Cu}$.

Fig. s8. $200 \mu \mathrm{m}$-sized AES maps of hBN/G bilayers.

Fig. s9. Core-level XPS spectra of $\mathrm{hBN} / \mathrm{G}$ bilayers.

Fig. s10. SEM images of $\mathrm{hBN} / \mathrm{G}$ bilayers on different $\mathrm{Cu}$ facets.

Fig. s11. Atomic resolution image and related FFT pattern of $\mathrm{hBN} / \mathrm{G}$ bilayers.

Fig. s12. Transfer curves of the devices of $\mathrm{hBN} / \mathrm{G}, \mathrm{SG}$, and $\mathrm{MG}$ samples measured in vacuum and air.

Fig. s13. Comparison of mobility and carrier concentration of graphene/hBN vertical heterostructure in this work and previous reports.

Fig. s14. Example of estimation of interlayer angle and lattice mismatch in hBN/G bilayers.

Tab. s1. Electrical performance of $\mathrm{MG}, \mathrm{SG}$, and $\mathrm{hBN} / \mathrm{G}$ bilayer on $\mathrm{SiO}_{2} / \mathrm{Si}$ substrate. 
a

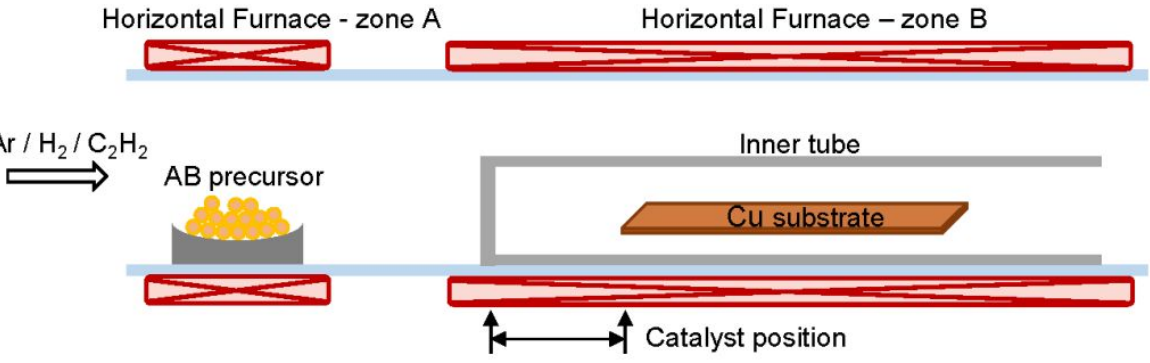

b

Quartz tube

Inner tube

Cu foil

Fig. s1. Schematic illustration of the furnace tube for the growth of $h B N / G$ bilayers.

(a) The position of $\mathrm{AB}$ precursor, inner tube, and $\mathrm{Cu}$ catalyst in the $\mathrm{CVD}$ chamber. The relative position between the inner tube and catalyst was fixed to reproduce the CVD growth. (b) Side-view of the positions of the CVD chamber tube, the inner tube, and the catalyst foil. 


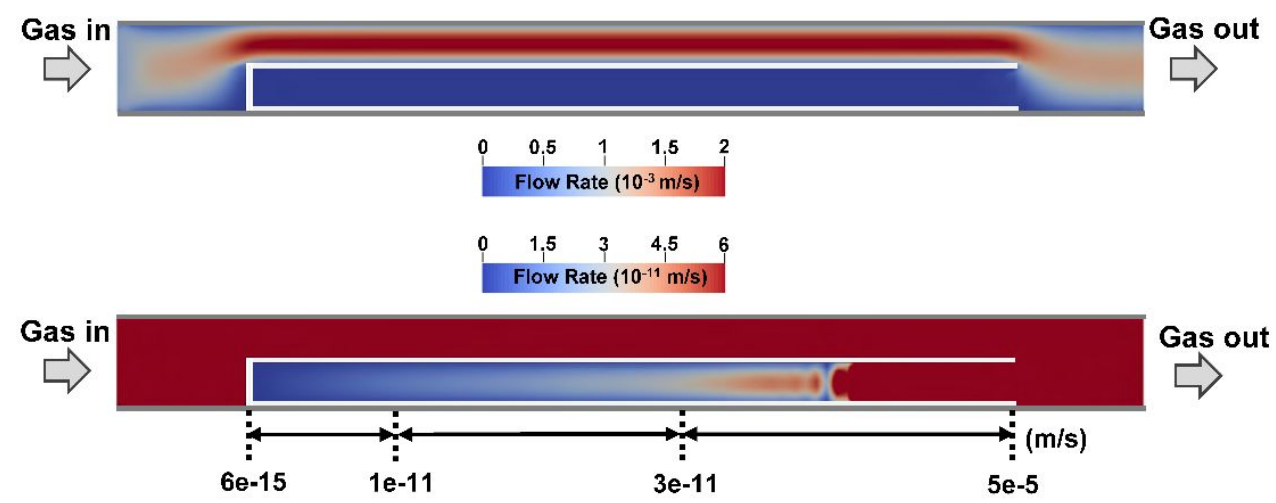

Fig. s2. Simulation of gas distribution in CVD furnace with a one-end-closed inner tube. These two images are the same simulation results presented in different ranges of flow rates. The sealed end of the inner tube is in the upstream direction, as mentioned in the description of the experiment. $40 \mathrm{sccm} \mathrm{H}_{2}$ was used here with a pressure of 0.9 Torr and a temperature of $1,000{ }^{\circ} \mathrm{C}$. The diameter of the inner tube is half of that of the outer tube, and the inner tube was placed in the middle position of the outer tube. The lengths of the inner and outer tubes are 300 and $400 \mathrm{~mm}$. The flow simulation shows that most of the hydrogen flowed directly through the outer tube. Only a small amount of hydrogen diffused into the inner tube. At the same time, the flow rate of hydrogen in the inner tube decreases continuously with the depth of the inner tube. As marked in the figure, at a distance of about 80 to $180 \mathrm{~mm}$ from the closed end, the hydrogen flow rate is slow and relatively stable in a range of $1 \times 10^{-11}$ to $3 \times 10^{-11} \mathrm{~m} / \mathrm{s}$. This is the range of $\mathrm{Cu}$ catalyst position we have chosen in the experiment. 


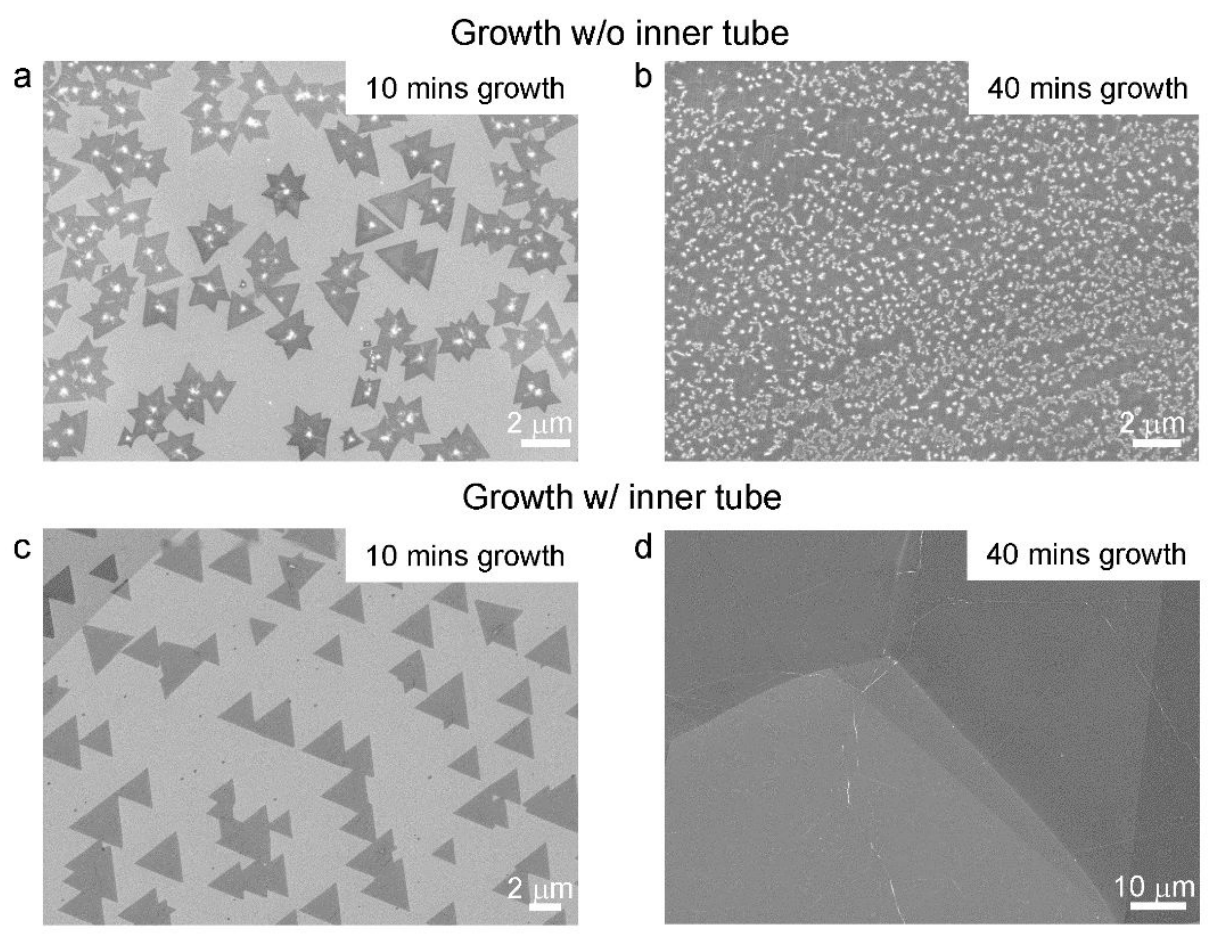

Fig. s3. SEM images of hBN grown with or without the inner tube. (a, c) 10 mins growth of hBN. (b, d) 40 mins growth of hBN. The application of the inner tube can greatly decrease the nucleation and growth rate of $\mathrm{hBN}$, and thus suppress the multilayer growth of hBN. 


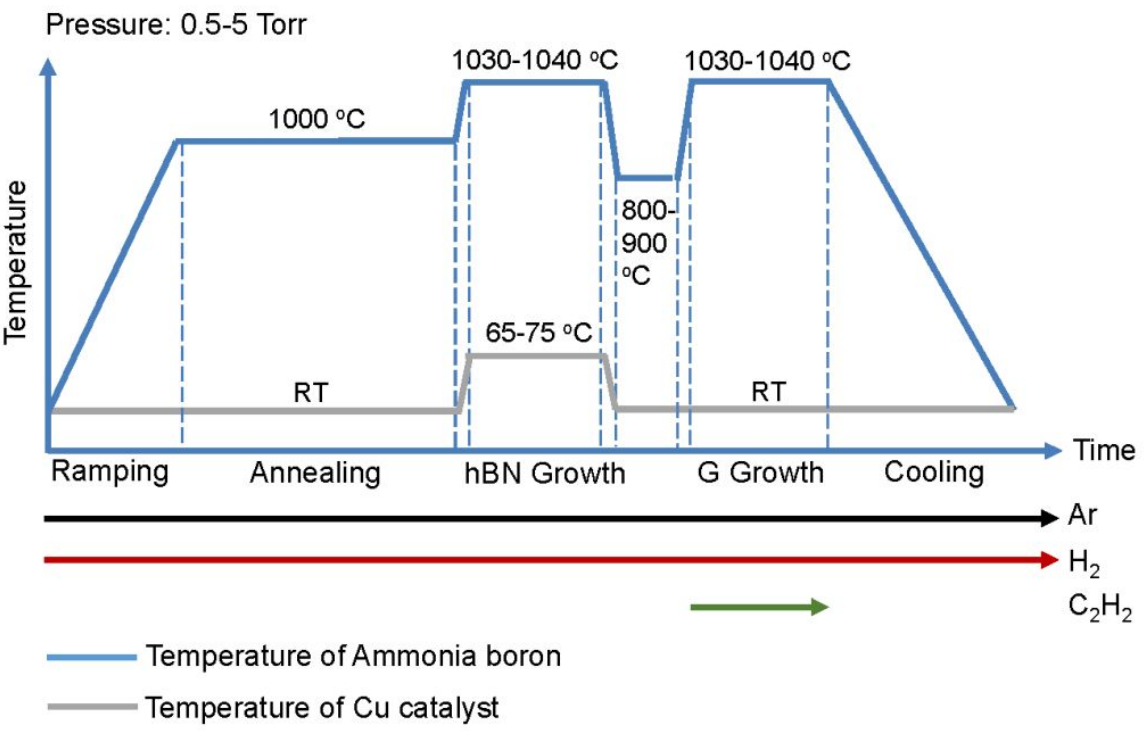

Fig. s4. Timeline of CVD growth of hBN/G on Cu catalyst. The whole CVD procedure was carried out at a pressure in the range of 0.5 to 5 Torr. 


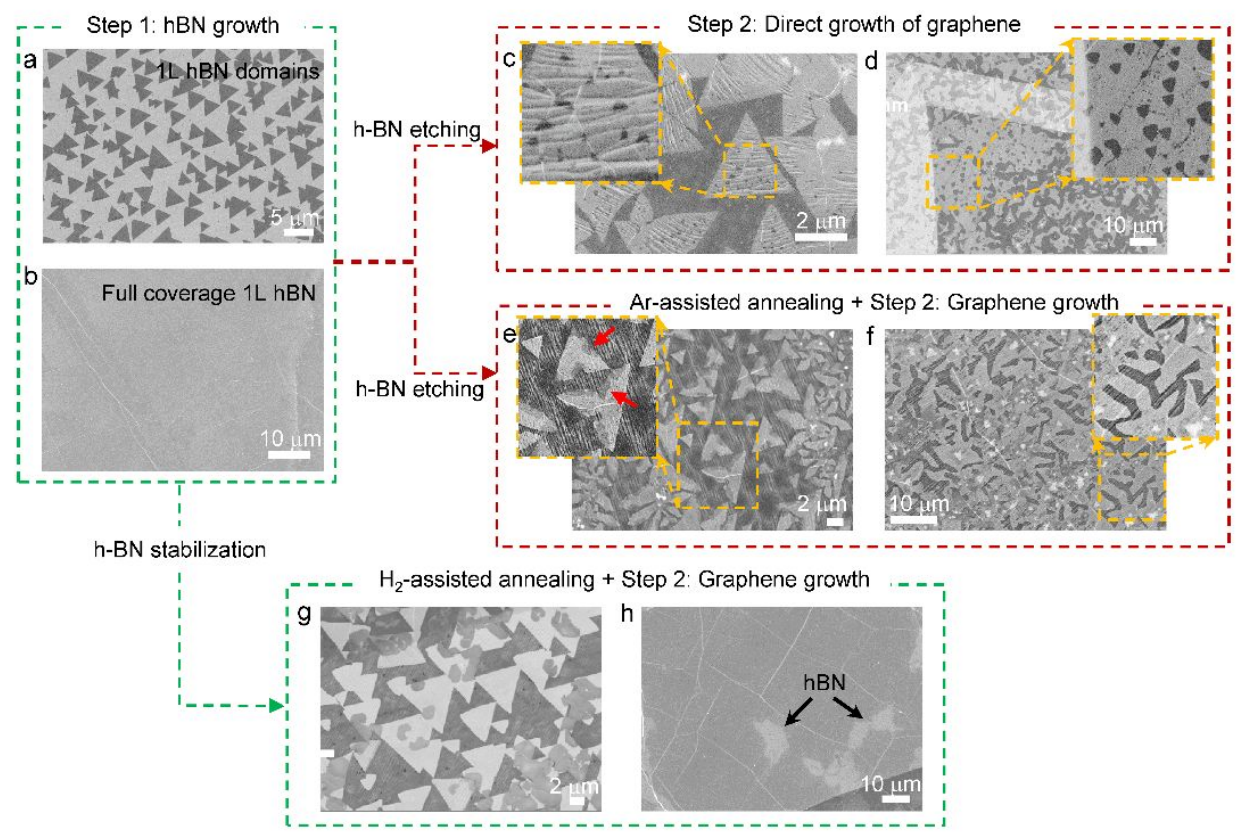

Fig. s5. SEM images of as-grown $h B N / G$ heterostructure film with different annealing steps. $(a, b) h B N$ templates. $(c, d)$ Direct growth of graphene after the growth of hBN. (e, f) Argon annealing between the growth of hBN and graphene. (g, h) Hydrogen annealing between the growth of $\mathrm{hBN}$ and graphene. The controlled experiments start with two types of hBN template: triangle domains, and full-coverage monolayer film. The direct growth of graphene following with the growth of hBN leads to the etching inside $\mathrm{hBN}$ domains and results in the formation of a lateral heterostructure of hBN/G. And for argon annealing between the growth steps of $\mathrm{hBN}$ and graphene, the etching was observed to happen at the edge of $\mathrm{hBN}$. Both lateral and vertical $\mathrm{hBN} / \mathrm{G}$ heterostructure was grown from the etched edge of hBN. Only the hydrogen annealing leads to a pure growth of the vertical bilayer heterostructure of $\mathrm{hBN} / \mathrm{G}$. 

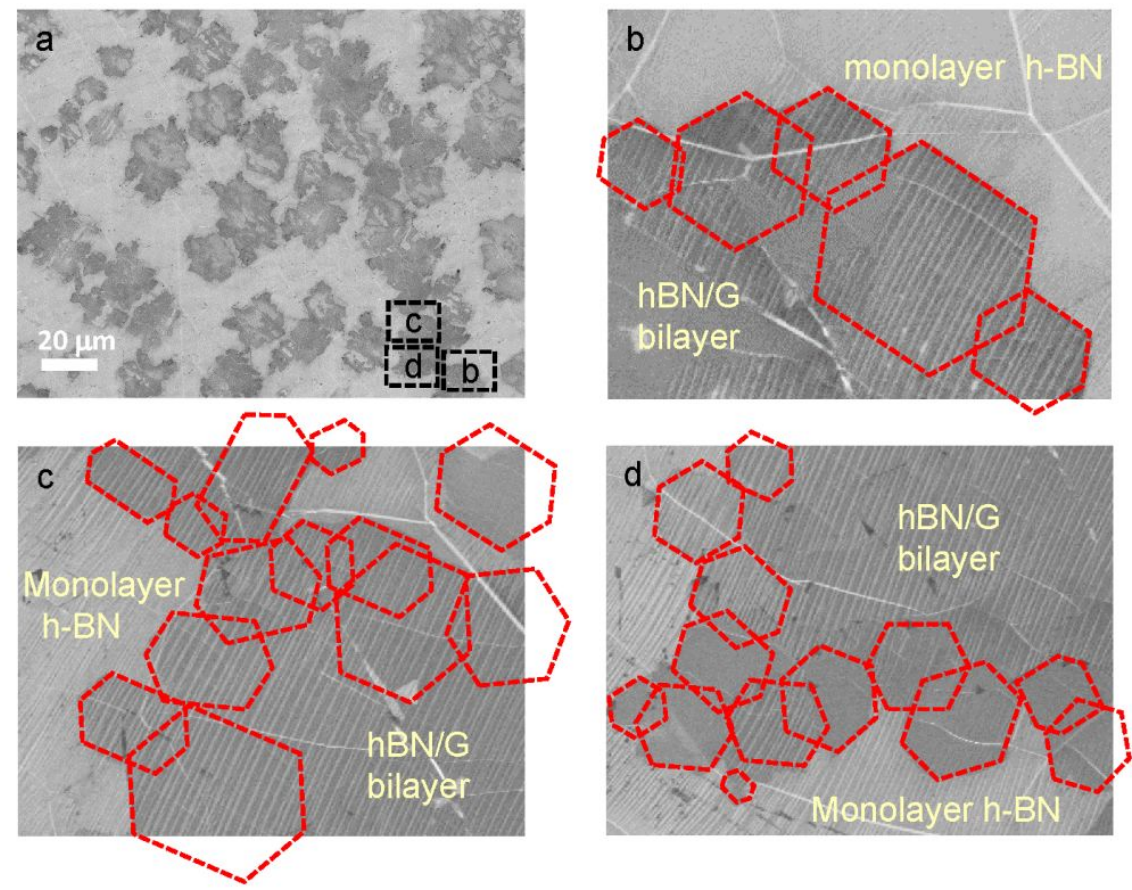

Fig. s6. SEM image of adlayer growth of graphene under full coverage hBN. (a) SEM image of $\mathrm{hBN} / \mathrm{G}$ bilayer on $\mathrm{Cu}$. The top $\mathrm{hBN}$ layer (lighter white color) has almost full coverage. And the lower layer graphene (darker grey color) was identified as islands formed by multiple hexagons. (b-d) Zoom-in views of the marked regions in (a). The red dashed hexagons were used to guide the graphene domains underneath the $\mathrm{hBN}$. The large graphene domain was observed to be composed of graphene hexagons. 


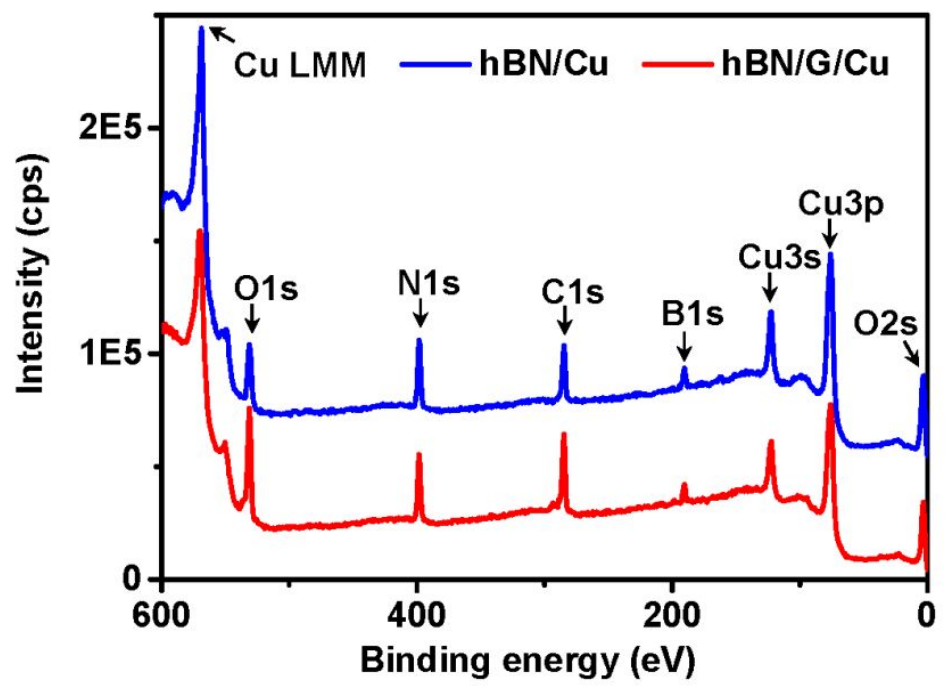

Fig. s7. XPS survey spectra of $\mathbf{h B N} / \mathrm{Cu}$ and $\mathbf{h B N} / \mathbf{G} / \mathbf{C u}$ samples. The curve of the $\mathrm{hBN} / \mathrm{Cu}$ was plotted with a Y offset of $50,000 \mathrm{cps}$ to clearly show the energy peaks. The presence of $\mathrm{C} 1 \mathrm{~s}$ and $\mathrm{O} 1 \mathrm{~s}$ peaks indicates that environment impurities from air (like $\mathrm{CO}_{2}$, $\mathrm{CO}, \mathrm{H}_{2} \mathrm{O}$, and $\mathrm{O}_{2}$ ) and $\mathrm{CVD}$ chamber (like $\mathrm{CuO}, \mathrm{Cu}_{2} \mathrm{O}$, amorphous carbon, and $\mathrm{CH}_{\mathrm{x}}$ ) would be adsorbed on the surface of the sample. 

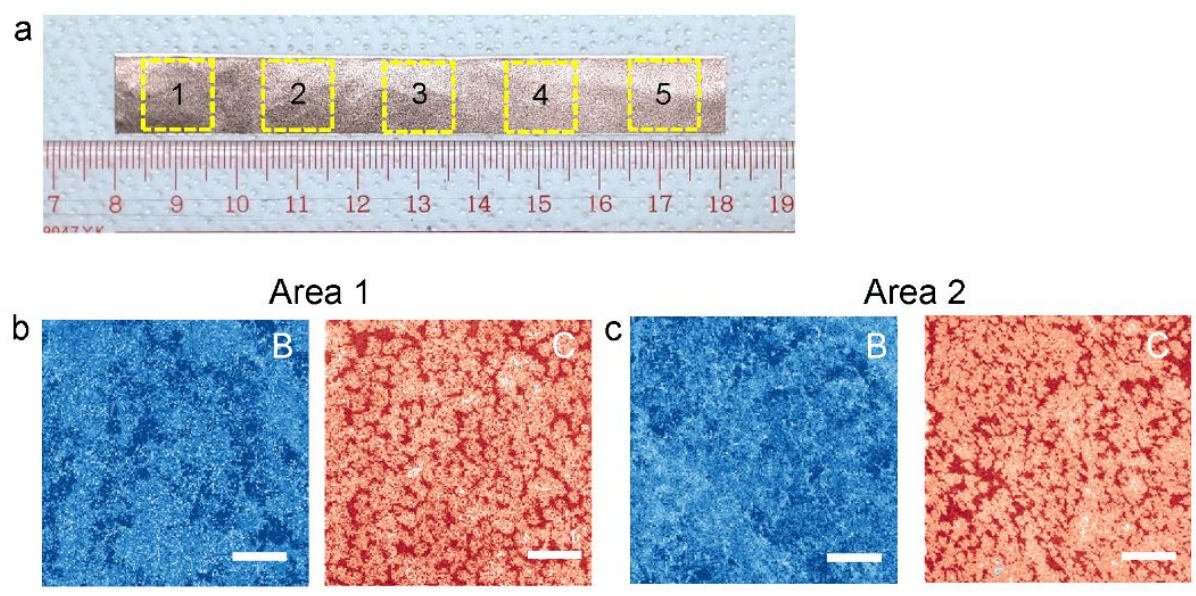

Area 3

Area 4
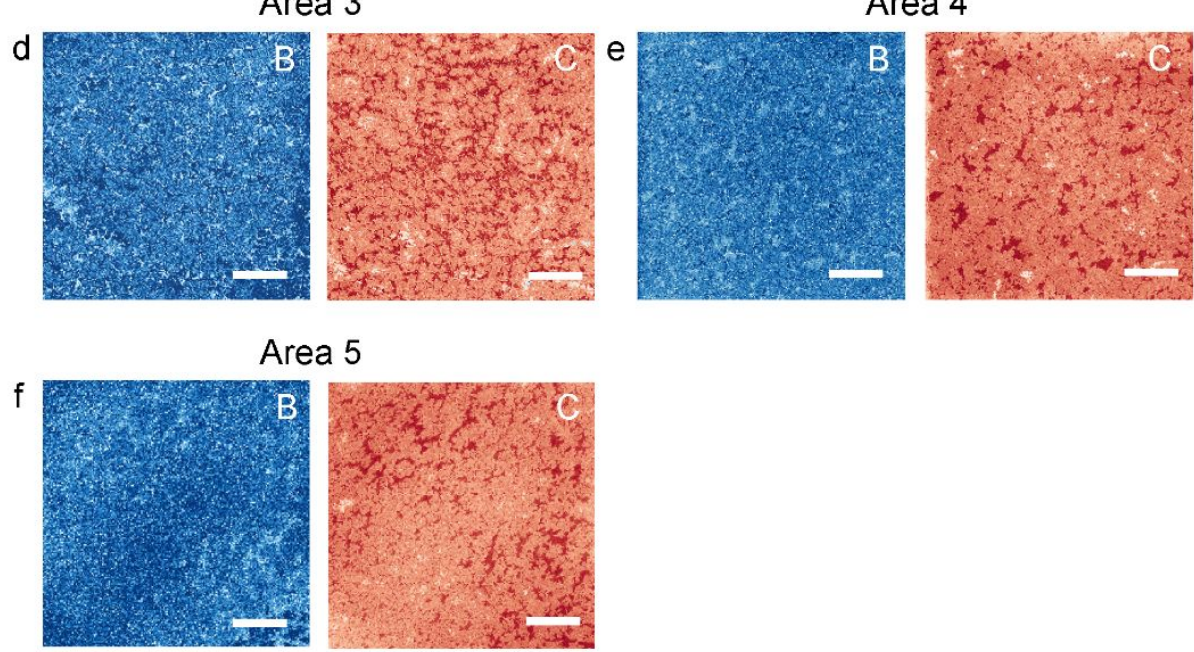

Fig. s8. $200 \mu \mathrm{m}$ AES maps of $\mathbf{h B N} / \mathbf{G}$ bilayers. (a) Optical image of the $\mathrm{hBN} / \mathrm{G} / \mathrm{Cu}$ sample shown in Fig. 2. The dashed squares mark the selected 5 areas for the following AES characterization. The direction from area 1 to area 5 is also the gas supply direction from upstream to downstream in the CVD growth. (b-f) AES B KLL and C KLL map of $\mathrm{hBN} / \mathrm{G}$ in Area 1 to Area 5. The scale bars are $40 \mu \mathrm{m}$. The homogeneous distribution of the B KLL map suggests that the hBN template remains intact in all the areas. And the estimation from the C KLL map shows that the graphene coverage varied from $70 \%$ (Area 1) to $92 \%$ (Area 5) along the direction of gas supply. 

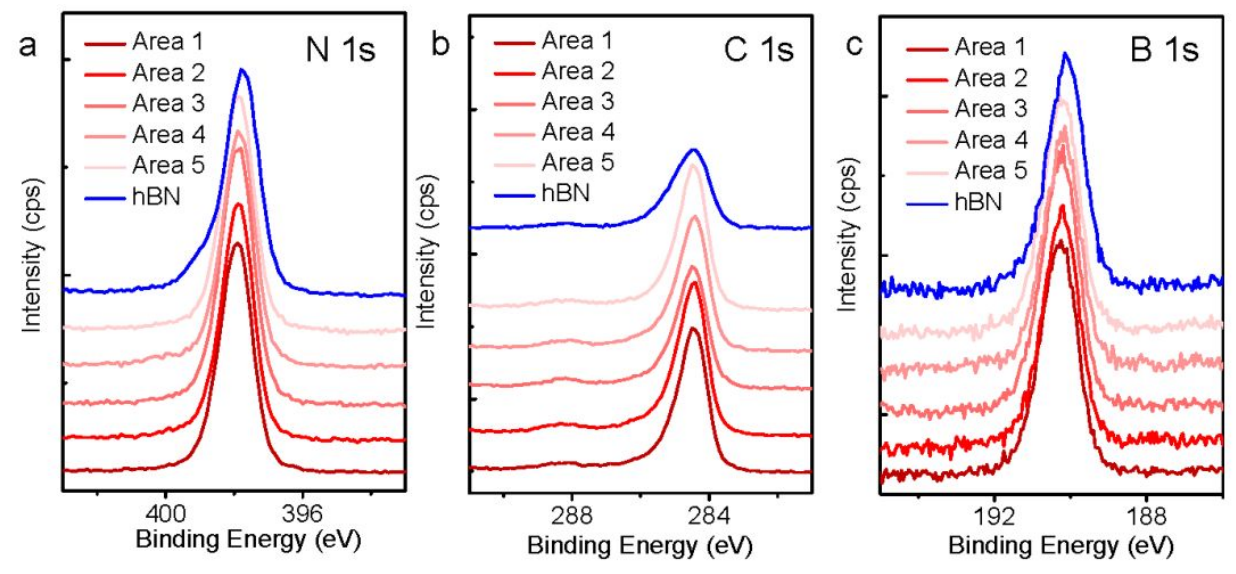

Fig. s9. Core-level XPS spectra of hBN/G bilayers. (a-c) N 1s, C 1s, and B 1s spectra of monolayer hBN and hBN/G bilayer in Area 1 to Area 5 marked in Fig. s7a. Both the peak energies and intensities of $\mathrm{N} 1 \mathrm{~s}$ and $\mathrm{B} 1 \mathrm{~s}$ in $\mathrm{hBN} / \mathrm{G}$ are in good agreement with monolayer $\mathrm{hBN}$, confirming the quality of $\mathrm{hBN}$ after graphene growth. The intensity of the $\mathrm{C}$ 1s peak is significantly larger in $\mathrm{hBN} / \mathrm{G}$ than monolayer $\mathrm{hBN}$, suggesting the presence of graphene in bilayer heterostructure. 

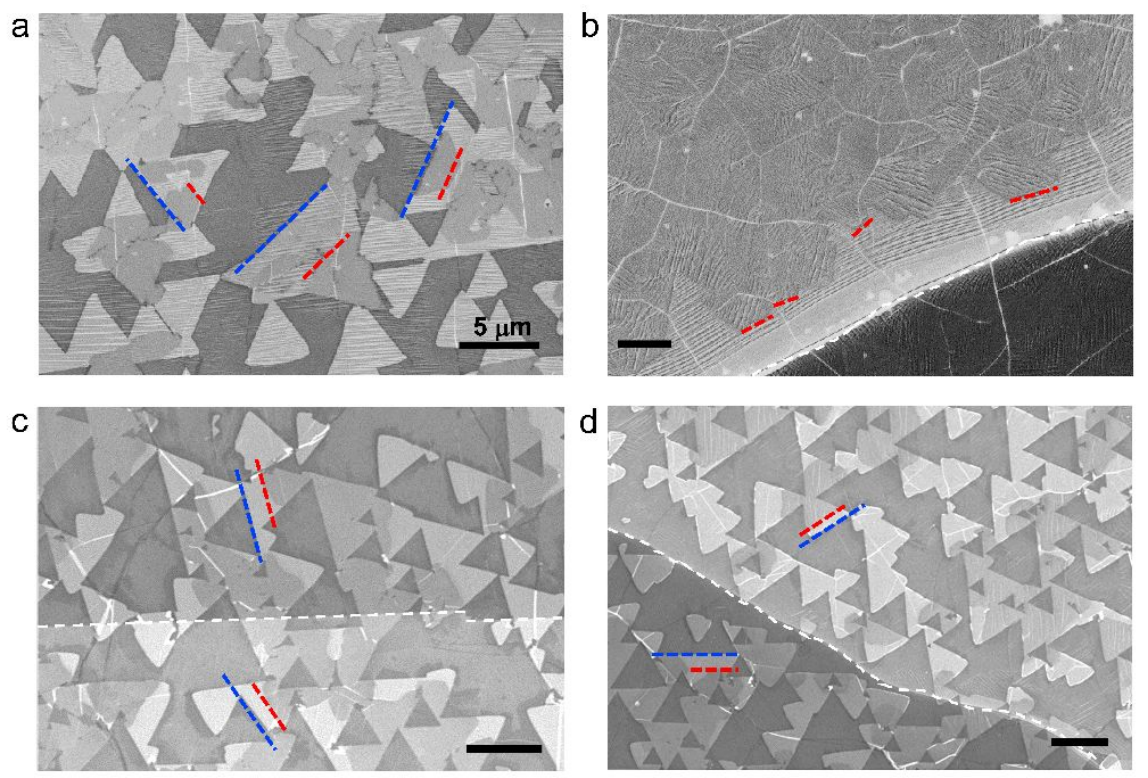

Fig. s10. SEM images of $\mathbf{h B N} / \mathbf{G}$ bilayer on different $\mathbf{C u}$ facets. $(\mathrm{a}, \mathrm{b}) \mathrm{hBN} / \mathrm{G}$ grown on one $\mathrm{Cu}$ grain. (c, d) hBN/G grown on different $\mathrm{Cu}$ grains. The red and blue dashed lines mark the orientation of bottom graphene and the orientation of the top $\mathrm{hBN}$ template, respectively When the $\mathrm{hBN}$ triangle has various orientations on the $\mathrm{Cu}$ grains, the crystal orientation of the bottom graphene is always consistent with the upper template (a, c, and d). When the hBN triangles are enlarged and connected to form a polycrystalline film, the underneath graphene is also formed as a corresponding polycrystalline film (b), as evident by the different directional hexagons at the edge of the underneath graphene layer. Therefore, the interlayer alignment between the hBN template and underlying graphene is independent of the hBN orientation. The scale bars are $5 \mu \mathrm{m}$. 


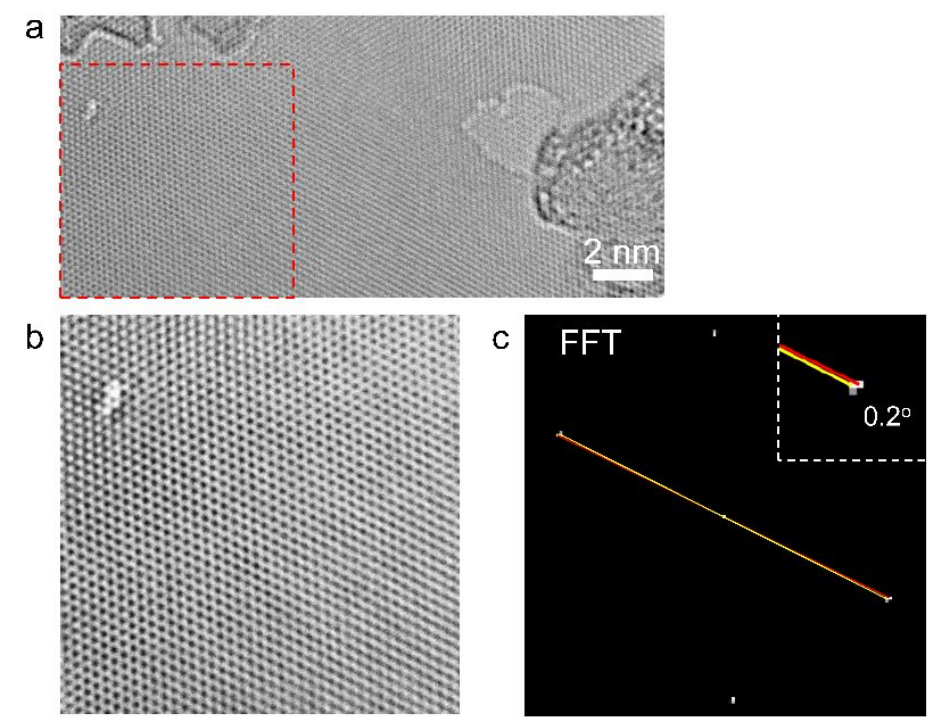

Fig. s11. Atomic resolution image and related FFT pattern of $h B N / G$ bilayers. (a) Bright-field TEM image of the $\mathrm{hBN} / \mathrm{G}$ bilayer film, which is a different region of the same sample used in Fig.4. (b) High-magnification image of hBN/G marked in (a). (c) The FFT pattern of the same hBN/G area in (b). A set of spots is marked with red (cross outer spots) and yellow (cross inner spots) lines. The interlayer angle was estimated to be $0.2^{\circ}$. 


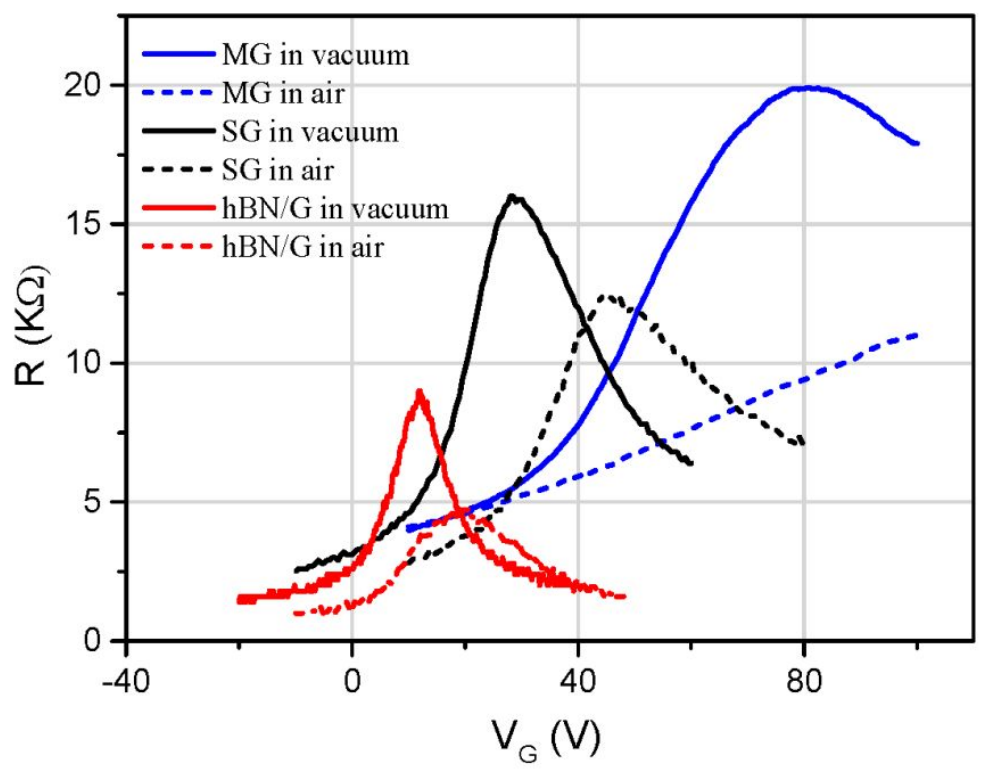

Fig. s12. Transfer curves of the devices of hBN/G, SG, and MG samples measured in vacuum and air. Compared with the electrical behaviors in a vacuum, all samples show higher $p$-doping levels in the air, as the adsorbates (such as water and oxygen) can act as $p$-type dopants on the surface device surface. The $\mathrm{hBN} / \mathrm{G}$ exhibits mobility of about $11,000 \mathrm{~cm}^{2} \mathrm{~V}^{-1} \mathrm{~s}^{-1}$ with a residue carrier concentration of $5.8 \times 10^{11} \mathrm{~cm}^{-2}$ in the air. The mobility of $\mathrm{hBN} / \mathrm{G}$ is almost twenty times (three times) of that of MG (SG), suggesting that $\mathrm{hBN}$ covered on top can largely improve the device performance of graphene. 


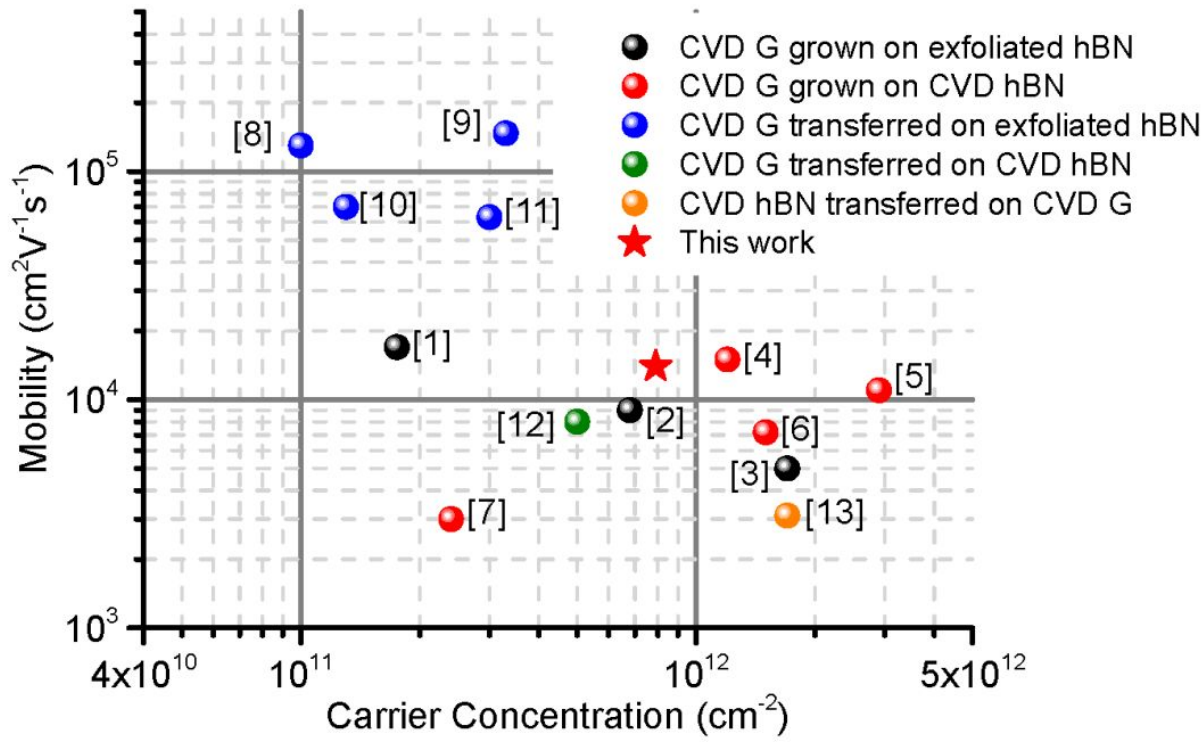

Fig. s13. Comparison of mobility and carrier concentration of graphene/hBN vertical heterostructure in this work and previous reports. ${ }^{1-13}$ Among these reports, (9), (2), and (3) are measured at $1.8 \mathrm{~K}, 1.5 \mathrm{~K}$, and $80 \mathrm{~K}$, respectively; the others are measured at room temperature. 

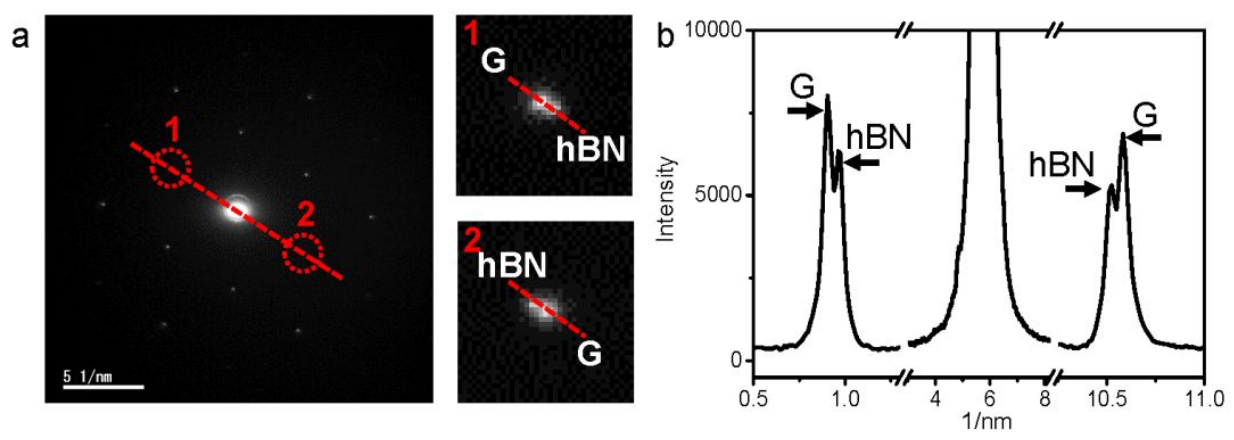

Fig. s14. Example of estimation of interlayer angle and lattice mismatch in hBN/G bilayers. (a) Diffraction pattern of $\mathrm{hBN} / \mathrm{G}$. A set of diffraction spots is marked in red circles, and it was selected to collect the interlayer angle and lattice mismatch. According to the intrinsic lattice constant, the outer spots are assigned to graphene, and the inner spots are assigned to $\mathrm{hBN}$. As guided by red dashed lines, the interlayer angle between graphene and $\mathrm{hBN}$ is $0^{\circ}$. (b) The cross-sectional profile of diffraction spots of $\mathrm{hBN} / \mathrm{G}$ marked in (a). Using the difference in the distance between two diffraction points from the same material, we can calculate the lattice mismatch between graphene and boron nitride. The lattice mismatch of $\mathrm{hBN}$ and graphene shown here is $0.7 \%$. 
Table s1. Statistical results of Dirac point voltage $\left(V_{D}\right)$, carrier density $\left(n_{0}\right)$, residual carrier density $\left(n_{\text {res }}\right)$, hole field-effect mobility $\left(\mu_{H o l e}\right)$, and electron field-effect mobility $\left(\mu_{\text {Electron }}\right)$ of monolayer graphene $(M G)$, single-crystalline graphene $(S G)$, and $h B N / G$ bilayer heterostructure on $\mathrm{SiO}_{2} / \mathrm{Si}$ substrate. The deviations list the maximum or minimum values in the electrical performance. The transport measurement was taken at room temperature.

\begin{tabular}{|c|c|c|c|c|}
\hline \multicolumn{2}{|c|}{ Sample information } & $M G$ & $S G$ & $h B N / G$ \\
\hline & $V_{D}(V)$ & $90( \pm 20)$ & $33( \pm 10.5)$ & $11( \pm 8)$ \\
\hline & $n_{0}\left(\mathrm{~cm}^{-2}\right)$ & $6.4( \pm 1.4) \times 10^{12}$ & $2.4( \pm 0.8) \times 10^{12}$ & $0.8( \pm 0.6) \times 10^{12}$ \\
\hline & res $\left(\mathrm{cm}^{-2}\right)$ & $3.0( \pm 0.8) \times 10^{12}$ & $3.8( \pm 1.2) \times 10^{11}$ & $2.4( \pm 1.0) \times 10^{11}$ \\
\hline$\mu_{\text {Hole }}$ & $@ n=5 \times 10^{11} \mathrm{~cm}^{-2}$ & $540( \pm 170)$ & $5700( \pm 1900)$ & $14650( \pm 6350)$ \\
\hline$\left(\mathrm{cm}^{2} V^{-1} s^{-1}\right)$ & $@ n=2 \times 10^{12} \mathrm{~cm}^{-2}$ & $600( \pm 320)$ & $4800( \pm 1400)$ & $11300( \pm 6350)$ \\
\hline$\mu_{\text {Electron }}$ & $@ n=5 \times 10^{11} \mathrm{~cm}^{-2}$ & $540( \pm 180)$ & $5400( \pm 1250)$ & $13500( \pm 4500)$ \\
\hline$\left(\mathrm{cm}^{2} V^{-1} s^{-1}\right)$ & $@ n=2 \times 10^{12} \mathrm{~cm}^{-2}$ & $N A$ & $2700( \pm 1100)$ & $8500( \pm 3200)$ \\
\hline
\end{tabular}




\section{References}

(1) Tang, S.; Wang, H.; Wang, H. S.; Sun, Q.; Zhang, X.; Cong, C.; Xie, H.; Liu, X.; Zhou, X.; Huang, F.; Chen, X.; Yu, T.; Ding, F.; Xie, X.; Jiang, M. SilaneCatalysed Fast Growth of Large Single-Crystalline Graphene on Hexagonal Boron Nitride. Nat. Commun. 2015, 6 (1), 6499. https://doi.org/10.1038/ncomms7499.

(2) Arjmandi-Tash, H.; Kalita, D.; Han, Z.; Othmen, R.; Nayak, G.; Berne, C.; Landers, J.; Watanabe, K.; Taniguchi, T.; Marty, L.; Coraux, J.; Bendiab, N.; Bouchiat, V. Large Scale Graphene/h-BN Heterostructures Obtained by Direct CVD Growth of Graphene Using High-Yield Proximity-Catalytic Process. J. Phys. Mater. 2018, 1 (1), 015003. https://doi.org/10.1088/2515-7639/aac66e.

(3) Yang, W.; Chen, G.; Shi, Z.; Liu, C.-C.; Zhang, L.; Xie, G.; Cheng, M.; Wang, D.; Yang, R.; Shi, D.; Watanabe, K.; Taniguchi, T.; Yao, Y.; Zhang, Y.; Zhang, G. Epitaxial Growth of Single-Domain Graphene on Hexagonal Boron Nitride. Nat. Mater. 2013, 12 (9), 792-797. https://doi.org/10.1038/nmat3695.

(4) Gao, T.; Song, X.; Du, H.; Nie, Y.; Chen, Y.; Ji, Q.; Sun, J.; Yang, Y.; Zhang, Y.; Liu, Z. Temperature-Triggered Chemical Switching Growth of In-Plane and Vertically Stacked Graphene-Boron Nitride Heterostructures. Nat. Commun. 2015, 6 (1), 6835. https://doi.org/10.1038/ncomms 7835.

(5) Song, X.; Gao, T.; Nie, Y.; Zhuang, J.; Sun, J.; Ma, D.; Shi, J.; Lin, Y.; Ding, F.; Zhang, Y.; Liu, Z. Seed-Assisted Growth of Single-Crystalline Patterned Graphene Domains on Hexagonal Boron Nitride by Chemical Vapor Deposition. Nano Lett. 2016, 16 (10), 6109-6116. https://doi.org/10.1021/acs.nanolett.6b02279.

(6) Wang, M.; Jang, S. K.; Jang, W.-J.; Kim, M.; Park, S.-Y.; Kim, S.-W.; Kahng, S.J.; Choi, J.-Y.; Ruoff, R. S.; Song, Y. J.; Lee, S. A Platform for Large-Scale Graphene Electronics - CVD Growth of Single-Layer Graphene on CVD-Grown Hexagonal Boron Nitride. Adv. Mater. 2013, 25 (19), 2746-2752. https://doi.org/10.1002/adma.201204904.

(7) Qian, Y.; Van Ngoc, H.; Kang, D. J. Growth of Graphene/h-BN Heterostructures on Recyclable Pt Foils by One-Batch Chemical Vapor Deposition. Sci. Rep. 2017, 7 (1), 17083. https://doi.org/10.1038/s41598-017-17432-9.

(8) Pezzini, S.; Mišeikis, V.; Pace, S.; Rossella, F.; Watanabe, K.; Taniguchi, T.; Coletti, C. High-Quality Electrical Transport Using Scalable CVD Graphene. 2D Mater. 2020, 7 (4), 041003. https://doi.org/10.1088/2053-1583/aba645.

(9) Banszerus, L.; Schmitz, M.; Engels, S.; Goldsche, M.; Watanabe, K.; Taniguchi, T.; Beschoten, B.; Stampfer, C. Ballistic Transport Exceeding $28 \mathrm{Mm}$ in CVD Grown Graphene. Nano Lett. 2016, $16 \quad$ (2), 1387-1391. https://doi.org/10.1021/acs.nanolett.5b04840. 
(10) De Fazio, D.; Purdie, D. G.; Ott, A. K.; Braeuninger-Weimer, P.; Khodkov, T.; Goossens, S.; Taniguchi, T.; Watanabe, K.; Livreri, P.; Koppens, F. H. L.; Hofmann, S.; Goykhman, I.; Ferrari, A. C.; Lombardo, A. High-Mobility, WetTransferred Graphene Grown by Chemical Vapor Deposition. ACS Nano 2019, 13 (8), 8926-8935. https://doi.org/10.1021/acsnano.9b02621.

(11) Banszerus, L.; Schmitz, M.; Engels, S.; Dauber, J.; Oellers, M.; Haupt, F.; Watanabe, K.; Taniguchi, T.; Beschoten, B.; Stampfer, C. Ultrahigh-Mobility Graphene Devices from Chemical Vapor Deposition on Reusable Copper. Sci. Adv. 2015, 1 (6), e1500222. https://doi.org/10.1126/sciadv.1500222.

(12) Qi, Z. J.; Hong, S. J.; Rodríguez-Manzo, J. A.; Kybert, N. J.; Gudibande, R.; Drndić, M.; Park, Y. W.; Johnson, A. T. C. Electronic Transport in Heterostructures of Chemical Vapor Deposited Graphene and Hexagonal Boron Nitride. Small 2015, 11 (12), 1402-1408. https://doi.org/10.1002/smll.201402543.

(13) Shautsova, V.; Gilbertson, A. M.; Black, N. C. G.; Maier, S. A.; Cohen, L. F. Hexagonal Boron Nitride Assisted Transfer and Encapsulation of Large Area CVD Graphene. Sci. Rep. 2016, 6 (1), 30210. https://doi.org/10.1038/srep30210. 